\title{
Affine transformations in an almost complex manifold with a natural affine connection
}

\author{
By Morio OBATA \\ (Received, April 12, 1956) \\ (Revised, July 12, 1956)
}

In an almost complex manifold there exists an affine connection in which the almost complex structure is covariant constant [2] ${ }^{1}$. In this paper such an affine connection, not necessarily symmetric, is said to be natural. When we speak of an almost complex manifold, we shall always bear a fixed natural affine connection in mind. An affine transformation in an affinely connected manifold is, roughly speaking, a differentiable transformation leaving the affine connection invariant $[6,12]$.

It might be of interest to ask whether an affine transformation preserves the almost complex structure or not, and if not, then what the structure of the manifold is. In this respect, A. Lichnerowicz [5] has recently proved that in an irreducible Kählerian manifold of dimension $2 n$ the largest connected group of isometries preserves the almost complex structure ${ }^{2)}$ if $n$ is odd or if $n$ is even and the Ricci curvature tensor does not vanish. J. A. Schouten and K. Yano [10] have also proved the same result for the pseudo-Kählerian manifold.

We shall prove that in an irreducible almost complex manifold if the largest connected group of affine transformations does not preserve the almost complex structure, then $n$ is even and the homogeneous holonomy group is contained in the real representation of the quaternionian linear group. Furthermore in this case a homomorphism of the group of all affine transformations into the special orthogonal group of three dimensions will be obtained. Our result generalizes the results of A. Lichnerowicz and J. A. Schouten and K. Yano.

In a complex manifold there exists, as is well-known, a symmetric

1) See the Bibliography at the end of the paper.

2) If the manifold is compact the theorem holds true without any other restriction [5]. 
natural affine connection [2] and an infinitesimal transformation preserves the complex structure if and only if it is complex analytic $[9,13]$. It therefore turns out that an infinitesimal affine transformation is always complex analytic in a complex manifold with symmetric natural affine connection whose homogeneous holonomy group is irreducible and is not contained in the real representation of the quaternionian linear group.

In $\S 1$ we shall make a brief sketch of the real representation of the complex matric group and then give a condition for a complex matric group to be a subgroup of the quaternionian linear group. In $\S 2$ we shall obtain the explicit form of the real matrix commuting with all elements of an irreducible real matric group, which is fundamental for our main theorem. $\S 3$ is concerned with the application of the preceding results to an almost complex manifold and the main theorem will be proved. In Appendix ${ }^{3)}$ we shall give an outline of the complex representation of the real matric group which is irreducible in the real number field but reducible in the complex number field.

\section{The quaternionian linear group}

Let $R$ be the field of real numbers and $C$ be that of complex numbers. We denote by $L(n, R)$ and by $L(n, C)$ the group of all regular matrices of degree $n$ with coefficients in $R$ and $C$ respectively.

Any element $A$ of $L(m, C)$ may be expressed in the form $A=A_{1}$ $+i A_{2}$ with real matrices $A_{1}, A_{2}$ of degree $m$. The correspondence

$$
A \rightarrow A^{\prime}=\left(\begin{array}{cc}
A_{1} & -A_{2} \\
A_{2} & A_{1}
\end{array}\right)
$$

gives an isomorphism of $L(m, C)$ with a subgroup $C L(m, R)$ of $L(2 m$, $R$ ). If $A$ is unitary, $A^{\prime}$ is orthogonal and vice versa. $C L(m, R)$ will be called the real representation of $L(m, C)$. It is easy to see that a matrix $A^{\prime} \in L(2 m, R)$ belongs to $C L(m, R)$ if and only if it commutes with $J_{m}, A^{\prime} J_{m}=J_{m} A^{\prime}$, where

3) This was added on a suggestion of the referee. The author wishes to express his gratitude to the referee whose suggestions and criticisms gave much improvement to the paper. 
Affine transformations in an almost complex manifold.

$$
J_{m}=\left(\begin{array}{lr}
0 & -E_{m} \\
E_{m} & 0
\end{array}\right)
$$

$E_{m}$ being the unit matrix of degree $m$. On putting

we have

$$
P=\frac{1}{\sqrt{ } 2}\left(\begin{array}{cc}
E_{m} & E_{m} \\
-i E_{m} & i E_{m}
\end{array}\right)
$$

$$
P^{-1} A^{\prime} P=\left(\begin{array}{cc}
A_{1}+i A_{2} & 0 \\
0 & A_{1}-i A_{2}
\end{array}\right)=\left(\begin{array}{cc}
A & 0 \\
0 & \bar{A}
\end{array}\right)
$$

and

$$
P{ }^{1} J_{m} P=\left(\begin{array}{cc}
i E_{m} & 0 \\
0 & -i E_{m}
\end{array}\right) \text {. }
$$

Thus $A^{\prime} \in L(2 m, R)$ belongs to $C L(m, R)$ if and only if $P^{-1} A^{\prime} P$ is of the form (1.1).

Next we denote by $\boldsymbol{Q}$ the algebra of quaternions and by $L(l, Q)$ the group of all regular matrices of degree $l$ with quaternion coefficients. Any element $A$ of $L(l, Q)$ may be written in the form $A=$ $A_{1}+j A_{2}$ with complex matrices $A_{1}, A_{2}$ of degree $l$, where $j$ is an element of the usual base $\{1, i, j, k\}$ of $Q$. We assign to $A$ the matrix $A^{\prime} \in L(2 l, C)$ defined by

$$
A^{\prime}=\left(\begin{array}{rr}
A_{1} & -A_{2} \\
\overline{A_{2}} & \overline{A_{1}}
\end{array}\right) .
$$

The correspondence $A \rightarrow A^{\prime}$ gives an isomorphism of $L(l, Q)$ with a subgroup $Q L(l, C)$ of $L(2 l, C) . \quad Q L(l, C)$ is called the quaternionian linear group. An easy computation shows

$$
A^{\prime} J_{l}=J_{l} \overline{A^{\prime}} \text {. }
$$

Conversely a matrix $A^{\prime}$ of $L(2 l, C)$ satisfying (1.3) is written in the form (1.2) and therefore belongs to $Q L(l, C)$. Thus $Q L(l, C)$ is the subgroup of $L(2 l, C)$ composed of all the matrices $A^{\prime}$ satisfying (1.3), Since $Q L(l, C) \subset L(2 l, C)$, the real representation $Q L(l, R)$ of $Q L(l, C)$ has the meaning by itself as a subgroup of $C L(2 l, R)$.

Now, let (S) be any subgroup of $Q L(l, C)$, then (S) is equivalent to 
its complex conjugate $\bar{\Im}$, because $\left[(1.3)\right.$ implies $J_{l}^{-1} A^{\prime} J_{l}=\overline{A^{\prime}}$ for all $A^{\prime}$ in (5). Conversely we have the following

PROPOSITION 1.4) Let (S) be a subgroup of $L(m, C)$ and $\overline{(S}$ its complex conjugate. We assume that $\mathbb{B S}$ is irreducible and is equivalent to $\overline{(S)}$ but is not equivalent to a subgroup of $L(m, R)$. Then we have:

1) There exists a matrix $S_{0} \in L(m, C)$ such that

$$
\begin{aligned}
& S_{0}^{-1} A S_{0}=\bar{A} \quad \text { for all } A \text { in } B \text { S. } \\
& \bar{S}_{0} S_{0}=\bar{S}_{0} S_{0}=-E_{m}
\end{aligned}
$$

and $S \in L(m, C)$ satisfies (1.4) if and only if $S$ is written in the from $S=\alpha S_{0}, \alpha$ being a non zero complex number. that

2) $m$ is even, $m=2 l$, and there exists a matrix $\lambda \in L(m, C)$ such

$$
\lambda^{-1} S_{0} \bar{\lambda}=J_{l}
$$

3) (3) is equivalent to a subgroup of $Q L(l, C)$.

To prove this we need the following lemmas.

LEMMA 1. If $S$ is a complex matrix satisfying $S \bar{S}=-E$, then there exists a regular matrix $\mu$ such that $S_{0}=\mu^{-1} S \bar{\mu}$ is unitary. Moreover if $S \bar{S}=E, S_{0}$ is symmetric, and if $S \bar{S}=-E, S_{0}$ is skew symmetric.

Proof. If we put $H=t \bar{S} S+E$, then $H$ is Hermitian positive definite. The condition $S \bar{S}= \pm E$ gives ${ }^{t} S H \bar{S}=\bar{H}$. Then there exists a unitary matrix $\mu_{1}$ such that ${ }^{t} \mu_{1} H_{\mu_{1}}$ is a diagonal matrix $H_{1}$. Since the coefficients of the diagonal of $H_{1}$ are real positive numbers, there exists a real diagonal matrix $\mu_{2}$ such that ${ }^{t} \mu_{2} H_{1} \mu_{2}=E$. On putting $\mu=\mu_{1} \mu_{2}$ we have ${ }^{t} \mu H_{\bar{\mu}}={ }^{t} \bar{\mu} \overline{\boldsymbol{H}} \mu=E$. It follows

$$
{ }^{t} \bar{S}_{0} S_{0}={ }^{t}\left(\bar{\mu}^{-1} \bar{S} \mu\right)\left({ }^{t} \bar{\mu} \bar{H} \mu\right)\left(\mu^{-1} S \bar{\mu}\right)={ }^{t} \mu H_{\mu}=E \text {, and }{ }^{t} S_{0} \bar{S}_{0}=E
$$

which shows that $S$ is unitary. We have also

$$
S_{0} \bar{S}_{0}=\left(\mu^{-1} S_{\mu}\right)\left(\bar{\mu}^{-1} \bar{S}_{\mu}\right)=\mu^{-1} S \bar{S}_{\mu}= \pm E .
$$

The conditions ${ }^{t} S_{0} \bar{S}_{0}=E$ and $S_{0} \bar{S}_{0}= \pm E$ imply $S_{0}= \pm{ }^{t} S_{0}$, which completes the proof of Lemma 1.

4) This has been proved by M. Abe [1] in a more generalized form using the theory of matric algebras. We shall give an elementary proof. 
The followings have been known [8].

LeMma 2. If $S_{0}$ is a symmetric matrix of degree $m$ with complex coefficients satisfying $S_{0} \bar{S}_{0}=E_{m}$, then there exists a unitary matrix $\nu$ such that $\nu^{-1} S_{0} \bar{\nu}=E_{m}$.

LEMMA 2'. If $S_{0}$ is a skew symmetric matrix of degree $m$ with complex coefficients satisfying $S_{0} \bar{S}_{0}=-E_{m}$, then $m$ is even, $m=2 l$, and there exists a unitary matrix $\nu$ such that $\nu^{-1} S_{0} \bar{\nu}=J_{l}$.

These allow us to prove Proposition 1.

PROOF OF PROPOSITION 1. By the assumed equivalence of 83 and $\overline{(3)}$ there exists a regular matrix $S_{1}$ such that

$$
S_{1}^{-1} A S_{1}=\bar{A} \quad \text { or } \quad \bar{S}_{1}^{-1} \bar{A} \bar{S}_{1}=A
$$

for all $A$ in (S), which implies $A\left(S_{1} \bar{S}_{1}\right)=\left(S_{1} \bar{S}_{1}\right) A$. Since $(B)$ is irreducible (in $C$ ), by Schur's lemma, $S_{1} \bar{S}_{1}$ is a numerical multiple of the unit matrix :

$$
S_{1} \bar{S}_{1}=\beta E_{m},
$$

$\beta$ being a non-zero complex number. (1.7) gives $\bar{S}_{1} S_{1}=\bar{\beta} E_{m}$, which together with (1.7) implies $\beta=\bar{\beta}$, i. e. $\beta$ is real. Then there exists a complex number $\beta_{0}$ such that $\beta_{0} \bar{\beta}_{0}=|\beta|$. On putting $\beta^{-1} S_{1}=S_{0}$ we have $S_{0}^{-1} A S_{0}=\bar{A}$ for all $A$ in $(S)$ and $S_{0} \bar{S}_{0}=\bar{S}_{0} S_{0}= \pm E_{m}$. In case $S_{0} \bar{S}_{0}=E_{m}$, by Lemma 1 and 2 there exists a regular matrix $\lambda_{0}$ such that $\lambda_{0}^{-1} S \bar{\lambda}_{0}$ $=E_{m}$. It follows that for any $A \in \mathbb{B S}$ we have

$$
\lambda_{0}^{-1} A \lambda_{0}=\left(\lambda_{0}^{-1} S_{0} \bar{\lambda}_{0}\right)^{-1}\left(\lambda_{0}^{-1} A \lambda_{0}\right)\left(\lambda_{0}^{-1} S_{0} \bar{\lambda}_{0}\right)=\bar{\lambda}_{0}^{-1}\left(S_{0}^{-1} A S_{0}\right) \bar{\lambda}_{0}=\bar{\lambda}_{0}^{-1} \bar{A} \bar{\lambda}_{0} .
$$

This means that $\lambda_{0}^{-1} A \lambda_{0} \in L(m, R)$ for any $A \in \mathbb{S}$, so that $(B)$ is equivalent to a subgroup of $L(m, R)$, contrary to the assumption.

Thus we have $S_{0} \bar{S}_{0}=-E_{m}$ and then by Lemma 1 and $2^{\prime}, m$ is even, $m=2 l$, and there exists a regular matrix $\lambda$ such that $\lambda^{-1} S_{0} \bar{\lambda}=J_{l}$. We have then for any $A \in B$

$$
\begin{aligned}
\left(\lambda^{-1} A \lambda\right) J_{l} & =\left(\lambda^{-1} A \lambda\right)\left(\lambda^{-1} S_{0} \bar{\lambda}\right)=\lambda^{-1} A S_{0} \bar{\lambda}=\lambda^{-1} S_{0} \bar{A} \bar{\lambda} \\
& =\left(\lambda^{-1} S_{0} \bar{\lambda}\right)(\bar{\lambda}-1 \bar{A} \bar{\lambda})=J_{l}(\bar{\lambda}-1 \bar{A} \bar{\lambda})
\end{aligned}
$$

This means that $\lambda^{-1} A \lambda \in Q L(l, C)$, so that $(B)$ is equivalent to a sub- 
group of $Q L(l, C)$.

Next let $S$ be any matrix satisfying (1.3). Then we have $S^{-1} A S$ $=S_{0}^{-1} A S_{0}$ for all $A \in \mathbb{B}$, from which we have $A\left(S S_{0}^{-1}\right)=\left(S S_{0}^{-1}\right) A$. (B) being irreducible, $S S_{0}^{-1}=\alpha E_{m}$, where $\alpha$ is a complex number. Thus we have $S=\alpha S_{0}$. Conversely if $S$ is written in the form $S=\alpha S_{0}$, then $S^{-1} A S=\bar{A}$ for all $A \in$ BS. Proposition 1 is thereby proved.

\section{The commutator algebra of the real irreducible matric group ${ }^{5)}$}

Let (S) be a subgroup of $L(n, R)$ acting on an $n$-dimensional real vector space $V$. A real matrix $K$ commuting with each element $A$ of (B), $K A=A K$, is called a commulator of $B$. The commutators of $(B)$ form an algebra $\AA$ of matrices, the commutator algebra. If $\mathbb{B}$ is irreducible (in $R$ ), then by Schur's lemma, any commutator $K$ of $\mathbb{B}$ is either zero or non-singular; in other words, the commutator algebra $\mathfrak{R}$ of $\mathbb{B}$ is a division algebra [11].

We assume that $\mathcal{B}$ is irreducible in $R$ but reducible in $C$. Then $n$ is even, $\boldsymbol{n}=\mathbf{2} m$, and we can find a base $\left\{\boldsymbol{u}_{1}, \cdots, \boldsymbol{u}_{m}, \overline{\boldsymbol{u}}_{1}, \cdots, \overline{\boldsymbol{u}}_{m}\right\}$ in $V^{c}$ relative to which every element $A$ of $B S$ has the form

$$
A=\left(\begin{array}{ll}
A_{1} & 0 \\
0 & \overline{A_{1}}
\end{array}\right) \text { with } A_{1} \in L(m, C),
$$

where $V^{c}$ denotes the complexification of $V$. Therefore $(B)$ is a subgroup of $C L(m, R)$. We denote by $\mathbb{B}_{1}$ the set of $A_{1}$ thus obtained, whose real representation is $\mathscr{G}$. Then $\mathscr{G}_{1}$ is irreducible in $C$, because $(B)$ is irreducible in $R$. If $K \in \Re$, then $K$ has the form

$$
K=\left(\begin{array}{ll}
K_{1} & K_{2} \\
\bar{K}_{2} & \bar{K}_{1}
\end{array}\right)
$$

where $K_{1}$ and $K_{2}$ are complex matrices of degree $m$, and we have

$$
\left(\begin{array}{cc}
A_{1} & 0 \\
0 & \overline{A_{1}}
\end{array}\right)\left(\begin{array}{ll}
K_{1} & K_{2} \\
\bar{K}_{2} & \overline{K_{1}}
\end{array}\right)=\left(\begin{array}{cc}
K_{1} & K_{2} \\
\bar{K}_{2} & \bar{K}_{1}
\end{array}\right)\left(\begin{array}{cc}
A_{1} & 0 \\
0 & \overline{A_{1}}
\end{array}\right)
$$

5) Concerning this section see Appendix, [1] and [11], Chap. III. 
If written down fully, this gives

$$
A_{1} K_{1}=K_{1} A_{1}, \quad A_{1} K_{2}=K_{2} \overline{A_{1}} \text {. }
$$

(S) $_{1}$ being irreducible, by Schur's lemma the first equality implies

$$
K_{1}=\alpha E_{m}
$$

$\alpha$ being a complex number. The second gives, again by Schur's lemma,

$$
K_{2}=0 \quad \text { or } \quad \operatorname{det} K_{2} \neq 0 \text {. }
$$

If $\operatorname{det} K_{2} \neq 0$, we have $K_{2}^{-1} A_{1} K_{2}=\bar{A}_{1}$ for all $A_{1} \in \mathbb{S}_{1}$. Since $\mathbb{S}_{1}$ can not be equivalent to a subgroup of $L(m, R)$, by Proposition 1, $m$ is even, $m=2 l$, and there exists a regular matrix $\lambda_{0}$ such that $\lambda_{0}^{-1} K_{2} \bar{\lambda}_{0}=$ $\beta J_{l}, \beta$ being a complex number. It should be noted that such $\lambda_{0}$ can be chosen independently of the special choice of $K$ in $\Omega$. On putting $\boldsymbol{v}_{\alpha}=\lambda_{0} \cdot \boldsymbol{u}_{\alpha},(1 \leqq \alpha \leqq m),\left\{\boldsymbol{v}_{1}, \cdots, \boldsymbol{v}_{m}, \overline{\boldsymbol{v}}_{1}, \cdots, \overline{\boldsymbol{v}}_{m}\right\}$ is a base in $V^{c}$ and relative to this base $K$ has the form

$$
K=\left(\begin{array}{ll}
\alpha E_{m} & \beta \boldsymbol{J}_{l} \\
\bar{\beta} J_{l} & \bar{\alpha} E_{m}
\end{array}\right) .
$$

If there exists $K$ such that $\operatorname{det} K_{2} \neq 0, \mathbb{S}_{1}$ is a subgroup of $Q L(l, C)$, $m=2 l$, and therefore (S) is a subgroup of $Q L(l, R)$. Conversely if (S) is a subgroup of $Q L(l, R)$, then matrices of the form (2.2) are commutators of (S).

Now we take the real base $\left\{\boldsymbol{f}_{1}, \cdots, \boldsymbol{f}_{m}, \boldsymbol{f}_{\overline{1}}, \cdots, \boldsymbol{f}_{\bar{m}}\right\}$ in $V^{c}$, therefore in $V$, which is related to $\left\{\boldsymbol{v}_{1}, \cdots, \boldsymbol{v}_{m}, \overline{\boldsymbol{v}}_{1}, \cdots, \overline{\boldsymbol{v}}_{m}\right\}$ by

$$
f_{\alpha}=\frac{1}{\sqrt{2 i}}\left(v_{\alpha}-\bar{v}_{\alpha}\right), \quad f_{\bar{\alpha}}=\frac{1}{\sqrt{2}}\left(v_{\alpha}+\bar{v}_{\alpha}\right)
$$

where $\alpha=1, \cdots, m$ and $\bar{\alpha}=\alpha+m$. Relative to this base every $A$ in (S) is written in the form

$$
A=\left(\begin{array}{cc}
B_{1} & -B_{2} \\
B_{2} & B_{1}
\end{array}\right),
$$

where $B_{1}$ and $B_{2}$ are real matrices of degree $m$ and are related to $A_{1}$ in (2.1) by $A_{1}=B_{1}+i B_{2}$. The matrices 


$$
E_{n},\left(\begin{array}{cc}
i E_{m} & 0 \\
0 & -i E_{m}
\end{array}\right),\left(\begin{array}{lr}
0 & J_{l} \\
J_{l} & 0
\end{array}\right),\left(\begin{array}{cc}
0 & i J_{l} \\
-i J_{l} & 0
\end{array}\right)
$$

relative to $\left\{\boldsymbol{v}_{\alpha}, \overline{\boldsymbol{v}}_{\alpha}\right\}$ are represented respectively by the real matrices

$$
E_{n},\left(\begin{array}{cc}
0 & -E_{m} \\
E_{m} & 0
\end{array}\right)=J_{m},\left(\begin{array}{cc}
J_{l} & 0 \\
0 & -J_{l}
\end{array}\right),\left(\begin{array}{cc}
0 & J_{l} \\
J_{l} & 0
\end{array}\right)
$$

relative to the base $\left\{\boldsymbol{f}_{\alpha}, \boldsymbol{f}_{\bar{\alpha}}\right\}$. They are clearly linearly independent in $R$. On putting $\alpha=a+i b, \beta=c+i d, a, b, c, d \in R$, we have

$$
K=a E_{n}+b J_{m} \text { or } K=a E_{n}+b J_{m}+c\left(\begin{array}{cc}
J_{l} & 0 \\
0 & -J_{l}
\end{array}\right)+d\left(\begin{array}{cc}
0 & J_{l} \\
J_{l} & 0
\end{array}\right)
$$

It follows that in case $(3)$ is a subgroup of $Q L(l, R)$ with $m=2 l, E_{n}, J_{m}$, $\left(\begin{array}{rr}J_{l} & 0 \\ 0 & -J_{l}\end{array}\right)$ and $\left(\begin{array}{rr}0 & J_{l} \\ J_{l} & 0\end{array}\right)$ form a base of $\Omega$ and in case $m$ is odd or $m=2 l$ and $(B)$ is not a subgroup of $Q L(l, R), E_{n}$ and $J_{m}$ form a base of $\Omega$.

PROPOSITION 2. Let (S) be'a subgroup of $L(n, R)$ acting on an $n$ dimensional real vector space $V$ and $\mathbb{R}$ the (real) commutator algebra of (S). We assume that (S) is irreducible in $R$ but reducible in $C$. Then $n$ is even, $n=2 m$, and (S) is a subgroup of $C L(m, R)$. If $m$ is even, $m=2 l$, and (S) is a subgroup of $Q L(l, R)$, then $E_{n}, J_{m},\left(\begin{array}{ll}J_{l} & 0 \\ 0 & -J_{l}\end{array}\right)$ and $\left(\begin{array}{cc}0 & J_{l} \\ J_{l} & 0\end{array}\right)$ form a base of $\AA$ relative to a suitable base in $V$. If $m$ is odd or if $m=2 l$ and (S) is not a subgroup of $Q L(l, R)$, then $E_{n}$ and $J_{m}$ form a base of $\Omega$ relative to a suitable base in $V$.

Next we denote by $\widetilde{\Re}$ the set consisting of all elements $K$ of $\Re$ such that $K^{2}=-E_{n}$. If $K=a E_{n}+b J_{m} \in \widetilde{\Re}$, we have $a^{2}-b^{2}=-1, a b=0$. If $a \neq 0, b$ must be 0 and then $a= \pm i$ contrary to the assumption that $a$ is real. Thus we have $a=0$ and $b= \pm 1$. Conversely $\pm J_{m}$ obviously belongs to $\widetilde{\Re}$. Thus in case $m$ is odd or $m$ is even, $m=2 l$, and $\mathscr{S}$ is not a subgroup of $Q L(l, R), \widetilde{\Omega}$ consists of $J_{m}$ and $-J_{m}$. 
If

$$
K=a E_{n}+b J_{m}+c\left(\begin{array}{rr}
J_{l} & 0 \\
0 & -J_{l}
\end{array}\right)+d\left(\begin{array}{ll}
0 & J_{l} \\
J_{l} & 0
\end{array}\right) \in \widetilde{\Re},
$$

we have $a^{2}-\left(b^{2}+c^{2}+d^{2}\right)=-1$ and $a b=a c=a d=0$. If $a \neq 0$, we would have $b=c=d=0$ hence $a^{2}=-1$ and wheih is impossible because $a$ must be real. Thus we have $a=0$ and $b^{2}+c^{2}+d^{2}=1$. Conversely $b J_{m}+c\left(\begin{array}{cc}J_{l} & 0 \\ 0 & -J_{l}\end{array}\right)+d\left(\begin{array}{rr}0 & J_{l} \\ J_{l} & 0\end{array}\right)$ with $b^{2}+c^{2}+d^{2}=1$ belongs to $\widetilde{\AA}$. Thus in case $\mathbb{B S}$ is a subgroup of $Q L(l, R)$ with $m=2 l, \widetilde{\Re}$ consists of the elements of the form $b J_{m}+c\left(\begin{array}{cc}J_{l} & 0 \\ 0 & -J_{l}\end{array}\right)+d\left(\begin{array}{rr}0 & J_{l} \\ J_{l} & 0\end{array}\right)$ with $b^{2}+c^{2}+d^{2}=1$.

Proposition 3. Notations and assumplions being as in Proposition 2 , let $\tilde{\Re}$ be the set consisting of all the elements $K$ of $\Re$ such that $K^{2}=-E_{n^{*}}$ Then if $(S)$ is a subgroup of $Q L(l, R)$ with $m=2 l, \widetilde{\AA}$ consists of the elements $K=b J_{m}+c\left(\begin{array}{cc}J_{l} & 0 \\ 0 & -J_{l}\end{array}\right)+d\left(\begin{array}{lr}0 & J_{l} \\ J_{l} & 0\end{array}\right)$ with $b^{2}+c^{2}+d^{2}=1$ relative to a suitable base in $V$. If $m$ is odd or if $m=2 l$ and $(5)$ is not a subgroup of $Q L(l, R), \widetilde{\Re}$ consists of $J_{m}$ and $-J_{m}$ relative to a suitable base in $V$.

\section{Affine transformations in an almost complex manifold}

Let $M$ be an $n$-dimensional affinely connected manifold of class $C^{\infty}$ and $\mathfrak{S}_{p}$ the homogeneous holonomy group of $M$ at a point $p$ of $M$. $\mathfrak{S}_{p}$ is a subgroup of $L(n, R)$ in the tangent space $T_{p}$ at $p . \quad M$ is called irreducible if $\mathfrak{S}_{p}$ is irreducible in $R$, i. e. if $\mathfrak{S}_{p}$ does not have any nontrivial invariant subspace of $T_{t}$. Otherwise, it is called reducible. This notion is independent of the choice of $p$, because a curve joining two points $p$ and $q$ determines, by parallel displacement, an isomorphism of $T_{p}$ onto $T_{q}$. Each parallel tensor field on $M$, i. e. a tensor field with null covariant derivative, induces on $T_{p}$ a tensor invariant under $\mathfrak{S}_{p}$. Conversely each tensor on $T_{p}$ invariant under $\mathfrak{S}_{p}$ deduces 
by parallel displacement a parallel tensor field.

We denote by $P(r, s)$ the set of all parallel tensor fields of type $(r, s)$ on $M$. If $\xi, \eta \in P(r, s)$ and $a, b \in R$, we have obviously $a \xi+b \eta$ $\in P(r, s)$. Hence $P(r, s)$ is a vector space over $R$. Since any element of $P(r, s)$ is uniquely determined by its value at a point $p, P(r, s)$ is isomorphic with the subspace of the tensor space of type $(r, s)$ over $T_{p}$ consisting of all tensors invariant under $\mathfrak{g}_{p}$. It follows that $P(r, s)$ is finite dimensional.

Now let $\varphi$ be a differentiable transformation of $M$ onto itself. We denote by the same letter $\varphi$ the differential mapping of $\varphi$, its extension to the tensor spaces and also that to the algebra of tensor fields. Denoting by $\nabla_{X}$ the covariant differentiation in the direction of a tangent vector $X, \varphi$ is called an affine transformation $[6,12]$ if $\varphi$ commutes with $\nabla_{X}$ for any $X$, i. e. $\varphi_{\nabla_{X}}=\nabla_{\varphi \cdot X} \varphi$. In case $\varphi$ is affine, if $F$ is a parallel tensor field, then so is $\varphi F$.

We denote by $A(M)$ the group of all affine transformations of $M$ onto itself, which is a Lie group with respect to the natural topology $[3,6] . \quad A_{0}(M)$ denotes the connected component of the identity in $A(M)$. If $\varphi \in A(M), \xi, \eta \in P(r, s)$ and $a, b \in R$, we have $\varphi(a \xi+b \eta)=$ $a(\varphi \cdot \xi)+b(\varphi \cdot \eta) \in P(r, s)$. Since there exists $\varphi^{-1} \in A(M), A(M)$ acts on $P(r, s)$ as a group of automorphisms. Thus we obtain a homomorphism $\rho$ of $A(M)$ into $L(h, R)$ defined by $\rho(\varphi) \cdot \xi=\varphi \cdot \xi$ for any $\xi \in P(r, s)$, where $h=\operatorname{dim} P(r, s) . \quad \rho$ is continuons.

An almost complex manifold is a manifold of class $C^{\infty}$ and of dimension even $2 m$ which has an almost complex structure $F$, i. e. which contains a tensor field $F=\left(F_{j}^{i}\right)$ of class $C^{\infty}$ satisfying

$$
F^{2}=-E \quad \text { or } \quad F_{j}^{i} F_{k}^{j}=-\delta_{k}^{i},
$$

where $i, j, k=1, \cdots, m, m+1, \cdots, 2 m$. It is always possible to define an affine connection, not necessarily symmetric, in which the almost complex structure $F$ is parallel [2]. Such an affine connection is called natural. When we speak of an almost complex manifold, we shall always keep a fixed naiural affine connection in mind. We can find a base in $T_{p}, p \in M$ such that relative to this base the tensor $F_{p}$ at $p$ has the form

$$
F_{p}=J_{m}
$$


Since $F_{p}$ is invariant under $\mathfrak{S}_{p}$, the matrix $F_{p}$ commutes with any element of $\mathfrak{g}_{p}$, so that any element $A$ of $\mathfrak{a}_{p}$ has the form

$$
A=\left(\begin{array}{cc}
A_{1} & -A_{2} \\
A_{2} & A_{1}
\end{array}\right)
$$

relative to the above base. This means that $\mathfrak{S}_{p}$ is a subgroup of $C L(m, R)$ and is reducible in $C$. A differentiable transformation of $M$ onto itself is said to preserve the almost complex structure $F$, if

$$
\varphi \cdot F=F \quad \text { or } \quad \varphi\left(F_{p}\right)=F_{\varphi \cdot p}
$$

for every point $p$ of $M$.

We assume in the sequel that the almost complex manifold $M$ is irreducible as an affinely connected manifold. Let $P(1,1)$ be the vector space spanned by all parallel tensor fields of type $(1,1)$ on $M$ and $\widetilde{P}(1,1)$ the subset of all the element $K$ of $P(1,1)$ such that $K^{2}=-E$ i. e. $K$ is an almost complex structure of $M$. Then any element $\varphi$ of $A(M)$ transforms linearly $\widetilde{P}(1,1)$ onto itself and further $\widetilde{P}(1,1)$ onto itself. Indeed, since the tensor field $E=\left(\delta_{j}^{i}\right)$ is invariant under all the transformations we have $(\rho(\varphi) \cdot K)^{2}=-\rho(\varphi) \cdot E=-E$ for every $K \in$ $\dot{P}(1,1)$. Assigning $K \in P(1,1)$ to the value $K_{p}$ of $K$ at $p, P(1,1)$ is isomorphic with the subspace of the tensor space of type $(1,1)$ over $T_{p}$ consisting of all tensors invariant under $\mathfrak{k}_{p}$, i. e. $P(1,1)$ is isomorphic with the commutator algebra $\mathfrak{K}$ of $\mathfrak{S}_{p}$. It is obvious that $\widetilde{P}(1,1)$ is isomorphic with the subset $\bar{\AA}$ of $\Omega$ consisting of the commutators $K$ such that $K^{2}=-E_{2 m}$.

Case I. $m$ is odd or $m$ is even, $m=2 l$, and $\mathfrak{S}_{p}$ is not a subgroup of $Q L(l, R)$. There exists, by Proposition 2, a base in $T_{p}$ such that relative to this base the tensor (3.2) has the same form and $\Omega$ is spanned by $E_{2 m}$ and $J_{m} . \quad E_{2 m}$ and $J_{m}$ deduce the parallel tensor field $E=\left(\delta_{j}^{i}\right)$ and the almost complex structure $F$. Thus $P(1,1)$ is spanned by $E$ and $F$. Since by Proposition $3 \hat{\Re}$ consists of $\pm J_{m}, \hat{P}(1,1)$ consists of $\pm F$. Since $\rho(\varphi) \cdot \widetilde{P}(1,1) \subset \widetilde{P}(1,1)$ for every $\varphi \in A(M)$, we have $\rho(\varphi) \cdot F= \pm F . \quad \rho$ being continuous, we have $\rho(\varphi) \cdot F=F$ for every $\varphi \in$ $A_{0}(M)$, i. e. $A_{0}(M)$ preserves the almost complex structure $F$.

Case II. $m$ is even, $m=2 l$, and $\mathfrak{S}_{p}$ is a subgroup of $Q L(l, R)$. 
There exists, by Proposition 2, a base in $T_{b}$ such that relative to this base the tensor (3.2) has the same form and $\Re$ is spanned by $E_{2 m}, J_{m}$, $\left(\begin{array}{rr}J_{l} & 0 \\ 0 & -J_{l}\end{array}\right)$ and $\left(\begin{array}{rr}0 & J_{l} \\ J_{l} & 0\end{array}\right)$. We denote by $G$ and $H$ the parallel tensor fields deduced from $\left(\begin{array}{rr}J_{l} & 0 \\ 0 & -J_{l}\end{array}\right)$ and $\left(\begin{array}{ll}0 & J_{l} \\ J_{l} & 0\end{array}\right)$ respectively. Then we have $G^{2}=-E, H^{2}=-E$ i. e. $F, G$ and $H$ are almost complex structures. Furthermore we have $F G=-G F=H, G H=-H G=F, H F=-F H=G$. Thus $P(1,1)$ has the structure of algebra, which is isomorphic to the algebra of quaternions. $A(M)$ acts on $P(1,1)$ not only as a group of automorphisms of the vector space but also as a group of automorphisms of the algebra. $\widetilde{P(1,1)}$ consists of the tensor field $K=a F+b G$ $+c H$ such that $a^{2}+b^{2}+c^{2}=1$. Since $\rho(\varphi) \cdot \widetilde{P}(1,1) \subset \widetilde{P}(1,1)$ for every $\varphi \in A(M)$, we have

$$
\begin{aligned}
& \rho(\varphi) \cdot \boldsymbol{F}=\boldsymbol{a}_{11} \boldsymbol{F}+\boldsymbol{a}_{21} \boldsymbol{G}+\boldsymbol{a}_{31} \boldsymbol{H} \in \widetilde{\boldsymbol{P}(1,1)} \\
& \rho(\varphi) \cdot \boldsymbol{G}=\boldsymbol{a}_{12} \boldsymbol{F}+\boldsymbol{a}_{22} \boldsymbol{G}+\boldsymbol{a}_{32} \boldsymbol{H} \in \widetilde{\boldsymbol{P}}(\mathbf{1}, \mathbf{1}) \\
& \rho(\varphi) \cdot \boldsymbol{H}=\boldsymbol{a}_{13} \boldsymbol{F}+\boldsymbol{a}_{23} \boldsymbol{G}+\boldsymbol{a}_{33} \boldsymbol{H} \in \widetilde{\boldsymbol{P}}(\mathbf{1}, \mathbf{1})
\end{aligned}
$$

where $a_{11}, \cdots, a_{33}$ are real numbers and $\sum_{i=1}^{3} a_{i j}^{2}=1, j=1,2,3$. We identify

$$
\rho(\varphi)=\left(\begin{array}{lll}
a_{11} & a_{12} & a_{13} \\
a_{21} & a_{22} & a_{23} \\
a_{31} & a_{32} & a_{33}
\end{array}\right) .
$$

Now, we denote by $P^{*}(1,1)$ the vector subspace of $P(1,1)$ spanned by $F, G$ and $H$. Then $\rho(\varphi)$, for every $\varphi \in A(M)$, is an automorphism of $P^{*}(1,1)$. If $\xi=\xi_{1} F+\xi_{2} G+\xi_{3} H$ and $\eta=\eta_{1} F+\eta_{2} G+\eta_{3} H$, where $\xi_{1}, \cdots, \eta_{3} \in R$, then we have

$$
\xi \bullet \eta=-\left(\xi_{1} \eta_{1}+\xi_{2} \eta_{2}+\xi_{3} \eta_{3}\right) E+\left(\xi_{2} \eta_{3}-\xi_{3} \eta_{2}\right) F+\left(\xi_{3} \eta_{1}-\xi_{1} \eta_{3}\right) G+\left(\xi_{1} \eta_{2}-\xi_{2} \eta_{1}\right) H,
$$

where $\xi_{1} \eta_{1}+\xi_{2} \eta_{2}+\xi_{3} \eta_{3}$ is the scalar product of $\xi$ and $\eta$ and is denoted by $(\xi, \eta) . \quad\left(\xi_{2} \eta_{3}-\xi_{3} \eta_{2}\right) F+\left(\xi_{3} \eta_{1}-\xi_{1} \eta_{3}\right) G+\left(\xi_{1} \eta_{2}-\xi_{2} \eta_{1}\right) H$ is called the vector product of $\xi$ and $\eta$ and is denoted by $\xi \times \eta$. Then we have

$$
\xi \cdot \eta=-(\xi, \eta) E+\xi \times \eta
$$


Since $\rho(\varphi) \cdot(\xi \cdot \eta)=(\rho(\varphi) \cdot \xi)(\rho(\varphi) \cdot \eta)$ for every $\varphi \in A(M)$, we have

$$
(\rho(\varphi) \cdot \xi, \rho(\varphi) \cdot \eta)=(\xi, \eta) \text { and }(\rho(\varphi) \cdot \xi) \times(\rho(\varphi) \cdot \eta)=\rho(\varphi)(\xi \times \eta) \cdot
$$

Thus the linear transformation $\rho(\varphi)$ leaves invariant the inner and vector products in $P^{*}(1,1)$, so that $\rho(\varphi)$ is orthogonal and

$$
\begin{aligned}
\operatorname{det} \rho(\varphi) & =((\rho(\varphi) \cdot F) \times(\rho(\varphi) \cdot \boldsymbol{G}), \rho(\varphi) \cdot \boldsymbol{H}) \\
& =(\rho(\varphi) \cdot(\boldsymbol{F} \times \boldsymbol{G}), \rho(\varphi) \cdot \boldsymbol{H})=(\boldsymbol{F} \times \boldsymbol{G}, \boldsymbol{H})=\mathbf{1} .
\end{aligned}
$$

Therefore $\rho(\varphi)$ is contained in the special orthognal group $S O(3)$ in $P^{*}(1,1)$.

Our study thus culminates in the following theorem

THEOREM 1. Let $M$ be an almost complex manifold of dimension $2 m$ with the almost complex structure $F$. We denote by $\mathfrak{S}_{p}, p \in M$, the homogeneous holonomy group of $M$ with respect to a natural affine connection. $A(M)$ denotes the group of all affine transformations of $M$ onto itself and $A_{0}(M)$ denotes the connected component of the identity of $A(M)$. We assume that $\mathfrak{S}_{p}$ is irreducible (in $R$ ). Then, $\mathfrak{S}_{p}$ is a subgroup of $C L(m, R)$. Further, (I) in case $m$ is odd or $m$ is even, $m=2 l$, and $\mathfrak{\mathfrak { Z }}_{p}$ is not a subgroup of $Q L(l, R)$, we have $\varphi \cdot F= \pm F$ for every $\varphi \in A(M)$. Especially we have $\varphi \cdot F=F$ for every $\varphi \in A_{0}(M)$, i. $e$. $A_{0}(M)$ preserves the almost complex structure. (II) In case $m$ is even, $m=2 l$ and $\mathfrak{S}_{p}$ is a subgroup of $Q L(l, R), M$ has three independent almost complex structure $F, G$ and $H$ such that $F G=-G F=H, G H=$ $-H G=F, H F=-F H=G$ and they are all parallel. $A(M)$ acts on the vector space spanned by $F, G$ and $H$ as a group of orthogonal transformations. Furthermore these orthogonal transformations belong to $S O(3)$ in the vector space.

COROLlARY 1. Nolations and assumplions being as in Theorem 1, if $m$ is odd or if $m=2 l$ and $\mathfrak{s}_{p}$ is not a subgroup of $Q L(l, R)$, then $A_{0}(M)$ preserves the almost complex structure.

COROLlary 2. Notations and assumplions being as in Theorem 1, if $A_{0}(M)$ does not preserve the almost complex structure $F$, then $m=2 l$ and $\mathfrak{S}_{p}$ is a subgroup of $Q L(l, R)$ and there exists a homomorphism of $A(M)$ into $S O(3)$.

We denote by $C(M)$ the group of all affine transformations preserving the almost complex structure $F$. Then $C(M)$ is a closed subgroup of $A(M)$. If $m$ is odd or if $m=2 l$ and $\mathfrak{s}_{p}$ is not a subgroup 
of $Q L(l, R)$, by Corollary 1 we have $A_{0}(M) \subset C(M) \subset A(M)$, so that we have $\operatorname{dim} C(M)=\operatorname{dim} A(M)$. In case $m=2 l$ and $\mathfrak{S}_{p}$ is a subgroup of $Q L(l, R), C^{*}(M)$ denotes the kernel of $\rho: A(M) \rightarrow S O(3)$, i. e. the group of all affine transformations preserving the almost complex structures $F, G$ and $H$. Then $C^{*}(M)$ is a closed normal subgroup of $A(M)$ and $C(M)$. By Theorem 1 (M)/C*(M) is isomorphic with a subgroup of $S O(3)$. Therefore we have

$$
\operatorname{dim} A(M)-\operatorname{dim} S O(3) \leqq \operatorname{dim} C^{*}(M) \leqq \operatorname{dim} C(M)
$$

so that we have $\operatorname{dim} A(M)-3 \leqq \operatorname{dim} C(M) \leqq \operatorname{dim} A(M)$. We have proved

COROLlARY 3. Nolations and assumptions being as in Theorem 1, and above, we have

$$
\operatorname{dim} A(M)-3 \leqq \operatorname{dim} C(M) \leqq \operatorname{dim} A(M) .
$$

A pseudo-Kählerian manifold $M$ of dimension $2 m$ is a Riemannian manifold which has an almost complex structure $F$ with null covariant derivative with respect to the Riemannian connection. If $M$ is pseudoKählerian, the homogeneous holonomy group is a subgroup of the real representation of the unitary group $U(m)$. In case $m=2 l, U(m) \cap$ $Q L(l, C)$ is nothing but the unitary symplectic group $S_{p}(l)$. As an immediate consequence of Theorem 1 we have

THEOREM 2. In an irreducible pseudo-Kählerian manifold $M$ of dimension $2 m$, if $m$ is odd or if $m=2 l$ and $\mathfrak{g}_{p}$ is not a subgroup of the real representation of $S_{p}(l)$, then $A_{0}(M)$ preserves the almost complex siructure.

. In a pseudo-Kählerian manifold, as is well-known [4], the Ricci curvature tensor vanishes if and only if $\mathfrak{d}_{p}$ is a subgroup of the real representation of the special orthogonal group $S U(m)$. Since $S_{p}(l)$ is a subgroup of $S U(2 l)$, if the Ricci curvature tensor does not vanish, then $\mathfrak{S}_{p}$ cannot be a subgroup of the real representation of $S_{p}(l)$. Thus we have

COROLLARY 4. In an irreducible pseudo-Kählerian manifold of dimension $2 m$, if $m$ is odd or if $m=2 l$ and the Ricci curvalure tensor does not vanish, $A_{0}(M)$ preserves the almost complex siructure; especially the largest connected group of isometries preserves the almost complex struciure. 
Now, in a complex manifold there exists a symmetric natural affine connection [2], so that when we speak of a complex manifold we shall always bear a fixed symmetric natural affine connection in mind. By a contravariant analytic vector field $[9,13]$ in a complex manifold, we shall mean a self-adjoint contravariant vector field $\left(\xi^{\alpha}, \overline{\xi^{\alpha}}\right)$ whose components are analytic functions of the complex coordinates. This condition is expressed by

$$
\xi_{; \beta}^{\alpha}=0 \quad \text { and } \quad \xi_{; \beta}^{\bar{\alpha}}=0,
$$

where the semi-colon denotes the covariant derivative with respect to the symmetric natural affine connection. This is also expressed by

$$
\xi_{; k}^{i} F_{j}^{k}-\xi_{; j}^{k} F_{k}^{i}=0
$$

in its real representation, or equivalently by the fact that the infinitesimal transformation $\xi^{i}$ preserves the almost complex structure $F$. Thus we have

THEOREM 3. In an irreducible complex manifold of dimension $2 m$, if $m$ is odd or if $m=2 l$ and the homogeneous holonomy group is not $a$ subgroup of $Q L(l, R)$, an infinitesimal affine transformation is always complex analytic.

COROLLARY 5. In an irreducible Kählerian manifold of dimension $2 m$, if $m$ is odd or if $m$ is even and the Ricci curvature tensor does not vanish, an infinitesimal affine iransformation is always complex analytic.

\section{Appendix}

Let $V$ be an $n$-dimensional real vector space and $V^{c}$ its complexification, i. e. the complex vector space deduced from $V$ by extension of the basic field. We can identify $V$ with a subset of $V^{c}$. Every base of $V$ is then a base of $V^{c}$. Let $P$ be a subspace of $V^{c}$ and $\boldsymbol{Q}$ a subspace of $V$. If $P \supset Q$, then $P \supset Q^{c}$.

Now let $a$ be an endomorphism of $V$. We denote by the same letter $a$ the endomorphism of $V^{c}$ extending $a$. If we select a base $\left\{\boldsymbol{e}_{1}, \cdots, \boldsymbol{e}_{n}\right\}$ in $V$, we may represent $a$ by a matrix $A=\left(a_{j}^{i}\right)$ of degree $n$ whose coefficients are given by $a \cdot \boldsymbol{e}_{j}=\sum_{i=1}^{n} \boldsymbol{a}_{j}^{i} \boldsymbol{e}_{i}, \boldsymbol{a}_{j}^{i} \in \boldsymbol{R}$. We shall adopt the convention that corresponding types like $a$ and $A$ are used to 
mark the transition from the endomorphism to the matrix. A vector $x=\sum_{i=1}^{n} x^{i} e_{i}$ is in $V$ if and only if $x^{i} \in R, 1 \leqq i \leqq n$. If $x=\sum_{i=1}^{n} x^{i} e_{i} \in$ $V^{c}, \bar{x}$ denotes the complex conjugate of $x$, i. e. $\bar{x}=\sum_{i=1}^{n} \bar{x}^{i} \boldsymbol{e}_{i}$. It is then easy to see $a \cdot x=a \cdot \bar{x}$ for any endomorphism $a$ of $V$.

Let (B) be a group of automorphisms of $V$. We assume $(B)$ is irreducible in $V$ (in $R$ ) but reducible in $V^{c}$ (in $C$ ). Then there exists an invariant subspace $P$ of $V^{c}$ such that $P \neq V^{c}, P \neq\{0\}$.

If we put $P^{\prime}=P \cap V$, then $P^{\prime}$ is a (real) subspace of $V$. Since $P$ and $V$ are both invariant under (S), so also is $P^{\prime}$. (3) being irreducible in $V, P^{\prime}=\{0\}$ or $P^{\prime}=V$. If $P^{\prime}=V$, we would have $P \supset P^{\prime c}=V^{c}$ contrary to the assumption $P \neq V^{c}$. Thus we have

(A.1) $P \cap V=\{0\}$ i.e. $P$ does not contain real vectors other than 0 . We denote by $\bar{P}$ the subspace of $P$ consisting of the complex conjugate of the vectors of $P$. Then $\bar{P}$ is clearly invariant under $\mathbb{S}$. On putting $\boldsymbol{Q}=\boldsymbol{P} \cap \bar{P}$ we have $\boldsymbol{Q}=\overline{\boldsymbol{Q}}$. Consequently, $\xi \in \boldsymbol{Q}$ implies $\bar{\xi} \in \boldsymbol{Q}$. Therefore we have $\xi+\bar{\xi} \in \boldsymbol{Q} \cap V, i(\xi-\bar{\xi}) \in \boldsymbol{Q} \cap V$. Since $\boldsymbol{Q} \cap V$ $\subset P \cap V$, by (A.1) $Q \cap V=\{0\}$ and therefore $\xi+\bar{\xi}=\xi-\bar{\xi}=0$. The last relation implies $\xi=0$. Thus we have

(A.2) $P \cap \bar{P}=\{0\}$.

Next, if we put $P+\bar{P}=S$. Since $P \neq\{0\}$, there exists a non zero $\xi$ in $P$. Then we have $\xi+\bar{\xi} \in S \cap V$. If $\xi+\bar{\xi}=0$, we would have $\bar{\xi} \in P$ and then by (A.2) we would have $\xi=0$, contrary to the fact $\xi \neq 0$. Thus we have $\xi+\bar{\xi} \neq 0$, which shows $S \cap V \neq\{0\} . \quad S \cap V$ being an invariant subspace of $V$, by the irreducibility of (S) we have $S \cap V=V$, which gives $S \supset V$. It follows $S \supset V^{c}$ and then $S=V^{c}$. Thus together with (A.2) we have

\section{(A.3) $P+\bar{P}=V^{c} \quad$ (direct sum).}

Now, let $U$ be a subspace of $P$ invariant under $(B$. Then $(U+\bar{U}) \cap V$ is a (real) subspace of $V$ invariant under (\$). By irreducibility of (B), $(U+\bar{U}) \cap V=\{0\}$ or $(U+\bar{U}) \cap V=V$. If $(U+\bar{U}) \cap V=\{0\}$ for any $\xi \in U$ we have $\xi+\bar{\xi}=i(\xi-\bar{\xi})=0$ and therefore $\xi=0$. This means $U=$ $\{0\}$. If $(U+\bar{U}) \cap V=V$, we have $U+\bar{U} \supset V^{c}$, which implies $U=P$. Thus we have

(A.4) (3) acts on P irreducibly, i.e. $P$ does not contain an invariant subspace other than $P$ itself and $\{0\}$. 
Let us denote by $m$ the complex dimension of $P$. Then we have $\boldsymbol{n}=\mathbf{2} m$ by (A.3). We select a base $\left\{\boldsymbol{v}_{1}, \cdots, \boldsymbol{v}_{m}\right\}$ in $P$, then $\left\{\overline{\boldsymbol{v}}_{1}, \cdots, \overline{\boldsymbol{v}}_{m}\right)$ is a base of $\overline{\boldsymbol{P}}$ and $\left\{\boldsymbol{v}_{1}, \cdots, \boldsymbol{v}_{m}, \overline{\boldsymbol{v}}_{1}, \cdots, \overline{\boldsymbol{v}}_{m}\right\}$ is a base of $V^{c}$. An easy computation shows

(A.5) $\xi \in V^{c}$ is in $V$ if and only if it is written in the form

$$
\xi=\sum_{\alpha=1}^{m} \bar{\xi}^{\alpha} \boldsymbol{v}_{\alpha}+\sum_{\alpha=1}^{m} \xi^{\alpha} \overline{\boldsymbol{v}}_{\alpha}, \quad \xi^{\alpha} \in \boldsymbol{C} .
$$

Let $k$ be an endomorphism of $V$. Then we have

$$
\begin{aligned}
& k \cdot \boldsymbol{v}_{\beta}=\sum_{\alpha=1}^{m} k_{\beta}^{\alpha} \boldsymbol{v}_{\alpha}+\sum_{\alpha=1}^{m} k_{\beta}^{\bar{\alpha}} \overrightarrow{\boldsymbol{v}}_{\alpha} \\
& k \cdot \overline{\boldsymbol{v}}_{\beta}=\sum_{\alpha=1}^{m} k^{\alpha}{ }_{\beta} \boldsymbol{v}_{\alpha}+\sum_{\alpha=1}^{m} k^{\alpha} \overline{\boldsymbol{v}}_{\alpha} \quad(\beta=\mathbf{1}, \cdots, m)
\end{aligned}
$$

where $k_{\beta}^{\alpha}, k_{\beta}^{\bar{\alpha}}, k_{\vec{\beta}_{\vec{\beta}}}^{\alpha}, k_{{ }_{\beta}}^{\bar{\alpha}_{\vec{\beta}}}$ are complex numbers. Since $k \cdot \boldsymbol{v}_{\beta}=k \cdot \overrightarrow{\boldsymbol{v}}_{\beta}$ we have $k^{\bar{\alpha}} \cdot \overline{k_{\beta}}$ and $k_{\beta}^{\bar{\alpha}}=\overline{k_{\beta^{\alpha}}}$. Thus $k$ is represented by the matrix

$$
K=\left(\begin{array}{cc}
K_{1} & K_{2} \\
\bar{K}_{2} & \bar{K}_{1}
\end{array}\right) \text { where } K_{1}=\left(k_{\beta}^{\alpha}\right), K_{2}=\left(k_{\vec{\beta}}^{\alpha}\right)
$$

Conversely an endomorphism $k$ of $V^{c}$ represented by a matrix of this form is an endomorphism of $V$. Especially every element $a$ of $(S)$ is represented by a matrix of the form

$$
A=\left(\begin{array}{ll}
A_{1} & 0 \\
0 & \overline{A_{1}}
\end{array}\right) \quad . A_{1} \in L(m, C)
$$

because $\boldsymbol{a}$ leaves $P$ and $\bar{P}$ invariant.

We summarize:

PROPOSITION. Let $V$ be an $n$-dimensional real vector space and $V^{c}$ its complexification. Let (B) be a group of automorphisms of $V$. We assume that $\mathbb{S}_{3}$ is irreducible in $R$ but reducible in $C$. Then $n$ is even, $\boldsymbol{n}=2 m$, and we can find a base $\left\{\boldsymbol{v}_{1}, \cdots, \boldsymbol{v}_{m}, \overline{\boldsymbol{v}}_{1}, \cdots, \overline{\boldsymbol{v}}_{m}\right\}$ in $V^{c}$ with the following properties:

1) An endomorphism of $V^{c}$ is that of $V$ if and only if it is represented by a matrix of the form 


$$
K=\left(\begin{array}{ll}
K_{1} & K_{2} \\
\bar{K}_{2} & \bar{K}_{1}
\end{array}\right)
$$

relative to this base.

2) Every $a \in \mathfrak{G}$ is represented by a matrix of the form

$$
A=\left(\begin{array}{ll}
A_{1} & 0 \\
0 & \overline{A_{1}}
\end{array}\right) \text { with } A_{1} \in L(m, C)
$$

relative to this base.

3) The set $\mathbb{B}_{1}$ of $A_{1}$ thus obtained is the subgroup of $L(m, C)$ whose real representation is $\mathbb{B S}$ and which is irreducible in $C$.

\section{Tokyo Metropolitan University}

\section{Bibliography}

[1] M. Abe, Irreduzibilität und absolute Irreduzibilität des Matrizensystemes, Proc. Phys. Math. Soc. Japan, 24 (1942), 769-789.

[2] A. Frölicher, Zur Differentialgeometrie der komplexen Strukturen, Math. Ann., 129 (1955), 50-95.

[3] J. Hano and A. Morimoto, Note on the group of affine transformations of an affinely connected manifold, Nagoya Math. J., 8 (1955), 85-95

[4] A. Lichnerowicz, Espaces homogènes kählériennes, Coll. Int. de Géom. Diff. Strasbourg, (1953), 171-184.

[5] A. Lichnerowicz, Sur les groupes d'automorphismes de certaines variétés kähleriennes, C. R. Acad. Sci. Paris, 239 (1954) 1344-1346.

[6] K. Nomizu, On the group of affine transformations of an affinely connected manifold, Proc. Amer. Math. Soc., 4 (1953), 816-823.

[7] K. Nomizu, Invariant affine connections on homogeneous spaces, Amer. J. Math., 76 (1954), 33-65.

[8] M. Obata, On subgroups of the orthogonal group, to appear.

[9] S. Sasaki and K. Yano, Pseudo-analytic vectors on pseudo-Kählerian manifolds, Pacific. J. Math., 5 (1955), 987-993.

[10] J. A. Schouten and K. Yano, On pseudo-Kählerian spaces admitting a continuous group of motions, Proc. Kon. Ned. Acad. Amst., 58 (Ind. Math, 17) (1955), 565-570.

[11] H. Weyl, The classical groups, Princeton, (1946).

[12] K. Yano, Groups of transformations in generalized spaces, Tokyo (1949).

[13] K. Yano and I. Mogi, On real representations of Kaehlerian manifolds, Ann. of Math., 61 (1955), 170-189. 